Brit. Heart f., 1967, 29, 120.

\title{
Histology, Extensibility, and Chemical Composition of Pulmonary Trunk in Persons Living at Sea-level and at High Altitude in Peru
}

\author{
Y. CASTILLO, H. KRUGER, J. ARIAS-STELLA^, A. HURTADO^, P. HARRIS†, \\ AND D. HEATH $\dagger$
}

From the High Altitudes Research Institute, Peruvian University of Medical and Biological Sciences, Lima, Peru, and the Departments of Medicine and Pathology, University of Birmingham

In the fœtus, the pressures in the pulmonary artery and aorta are of the same order, and the structure of the pulmonary trunk is similar to that of the aorta (Heath et al., 1959). Both vessels show the same dense network of elongated and parallel elastic fibres (Heath et al., 1959). By the end of the first month of extrauterine life the pulmonary arterial pressure has fallen to a value that remains approximately the same throughout the rest of life. During the first two years of extrauterine life, the elastic tissue in the wall of the pulmonary trunk undergoes involution, becoming fragmented and subsequently forming an open network of branched fibrils (Heath et al., 1959).

The tissue of the media of the normal adult pulmonary trunk shows a greater degree of extensibility than does that of the aorta (Harris and Heath, 1962; Harris, Heath, and Apostolopoulos, 1965a). This may be due to the lower proportion of elastin in the pulmonary trunk, but differences in the proportion of collagen may also play a part. Throughout life, there is a progressive decrease in the extensibility of the tissue of the pulmonary trunk, and this has been ascribed to the apparent increase in the proportion of collagen visible under the microscope (Harris et al., 1965a).

When pulmonary hypertension has persisted from birth, as a result of a large congenital posttricuspid shunt, the elastic tissue in the wall of the pulmonary trunk preserves its fœtal appearance and remains similar to that in the aorta (Heath et al.,

Received April 5, 1966.

* Supported by United States Public Health Service Research Grants HE 07000-03 and RG 857604.

† Supported by a grant from the Wellcome Trust.
1959). The extensibility of the tissue of the pulmonary trunk is less than normal in such patients (Harris, Heath, and Apostolopoulos, 1965b). By contrast, when pulmonary hypertension arises later in life, as a result of a pre-tricuspid shunt or mitral stenosis, the elastic tissue of the pulmonary trunk remains of the normal adult type (Heath et al., 1959), and the extensibility of the wall is normal (Harris et al., 1965b).

People native to high altitudes sustain a permanently high pressure in the pulmonary artery (Peñaloza et al., 1962), and the appearance of the elastic tissue in the wall of the pulmonary trunk may resemble that in the aorta (Saldaña and AriasStella, 1963). The present study has been designed to investigate the extensibility of the pulmonary trunk in people living at sea-level and at high altitude in Peru, and to compare this physical property with the microscopical structure and with the chemicallydetermined proportions of elastin and collagen.

\section{Materials and Methods}

Specimens of pulmonary trunk were removed after death from 73 subjects. Sixty-six had died in Lima, which is at sea-level, but some of these had lived most of their lives at a higher altitudeand had died in Lima during transit through the city, or had been resident in Lima for only a short time before their death. Seven had lived and died in the vicinity of Cerro de Pasco, which is at an altitude of 14,200 feet.

The adventitia was removed from the pulmonary trunk and the extensibility of a circumferential strip measured by the method previously described (Harris et al., 1965a). On this occasion the volume of the arterial strip was assessed by weighing it and assuming that the specific gravity was approximately unity. The 120 
the volume by the length. A series of extensile loads up to $100 \mathrm{~g}$. were employed and the results expressed as the percentage extension for a given extensile force (dynes/mm. ${ }^{2}$ cross-sectional area).

An adjacent piece of the pulmonary trunk was removed for histological examination. It was embedded in paraffin wax in the usual way, and transverse sections were prepared so that the microtome knife cut the long axis of the strip in order to minimize distortion. Thin sections $(5 \mu)$ were stained by the Lawson modification of the Weigert-Sheridan method for elastic tissue and were counterstained with van Gieson's reagents to demonstrate collagen and muscle fibres.

The remainder of the pulmonary trunk (from which the adventitia had been removed) was cut into small pieces by scissors and placed in a vacuum desiccator. After drying, the material was ground with a mortar and pestle and returned to the desiccator to await chemical analysis. Collagen and elastin were estimated in this dried material using the method of Lowry, Gilligan, and Katersky, 1941. Two minor modifications were made to the method. The first extraction with $0.1 \mathrm{~N} \mathrm{NaOH}$ was prolonged to 48 hours, since this was found to increase the removal of soluble material by an average of $1 \cdot 1$ per cent of the original dry weight in a preliminary series of four duplicate analyses. Autoclaving was carried out at a pressure of $15 \mathrm{lb}$./sq. in. for 54 hours. Preliminary observations on a series of four replicated specimens showed that the average quantity of collagen extracted was $23 \cdot 7$ per cent of the dry weight after 32 hours of autoclaving, 24.8 per cent after 48 hours, and 25.3 per cent after 64 hours. Measurements of collagen were made on 31 specimens and measurements of elastin on 45 specimens.

\section{RESULTS}

Histology. The pattern of the elastic fibrils in the media of the pulmonary trunks of the subjects studied was found to be one of three main types which we have previously designated "adult pulmonary", "aortic", and "transitional" (Heath et al., 1959; Saldaña and Arias-Stella, 1963).

Of the 66 subjects who had died in Lima, 63 showed the adult pulmonary configuration of elastica in their pulmonary trunk (Fig. 1). This pattern also occurred in 4 of the 7 who had lived all their lives at high altitude and had died at Cerro de Pasco (Fig. 1). In this pattern the elastic tissue was in the form of an open network of branched, irregularly-shaped fibrils which sometimes showed clubbed terminations. The fibrils were not regular and did not run parallel with one another. While the elastic tissue was much less dense than in the media of the aorta or in the media of the pulmonary trunk showing an aortic configuration of elastica, it could only rarely be described as being sparse in amount. Furthermore, there was a considerable variation in the amount of elastic tissue present, so that in some cases the network of fibrils

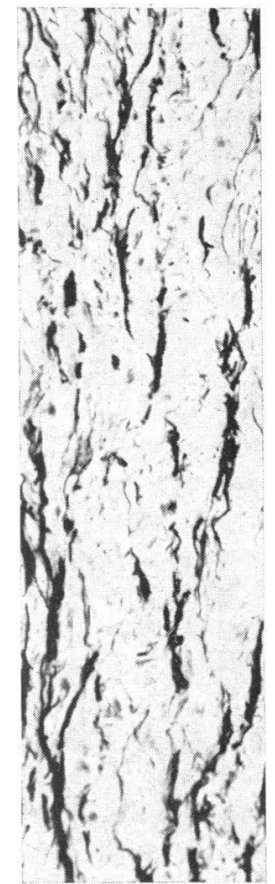

Fig. 1.-Transverse section of the pulmonary trunk from a Peruvian Indian aged 14 years who was born and spent his entire life at high altitude in the Andes. There is a normal adult pulmonary pattern of elastic tissue. (Elastic-van Gieson. $\times$ 150.)

was quite dense. This variation in the density of the elastica in the media of the normal pulmonary trunk has been pointed out before (Wagenvoort, Heath, and Edwards, 1964), and it is essential to bear this considerable normal variation in mind when studying sections of the pulmonary trunk, so that an unusually dense adult pulmonary pattern of elastica is not erroneously regarded as being of aortic type. In general, the elastic tissue pattern of the media of the pulmonary trunk of Peruvians resident at sea-level showed the same general appearance and variation as that which had previously been found in people resident in Birmingham in Great Britain (Harris et al., 1965a). It was frequently possible to distinguish between the pulmonary trunks of young and old subjects, the age of distinction being somewhere in the region of $\mathbf{4 0}$ years. In general the elastic fibrils of subjects below that age were clearly delineated from the surrounding fibro-muscular tissue and stood out sharply in sections stained by the elastic-van Gieson method. In contrast, the elastic fibrils of subjects beyond that age showed a certain fuzziness of outline, with the presence of very fine connecting fibrils forming a background elastosis; in the older 


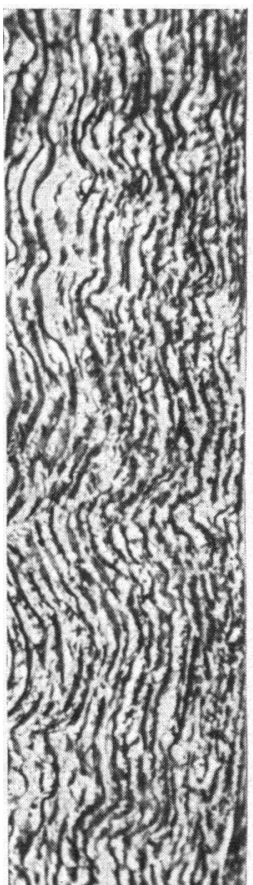

(A)

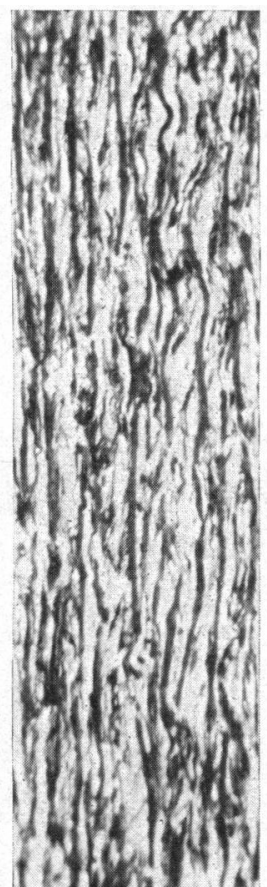

(B)

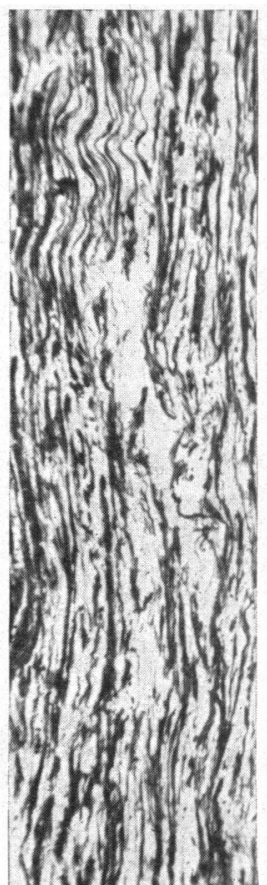

(C)

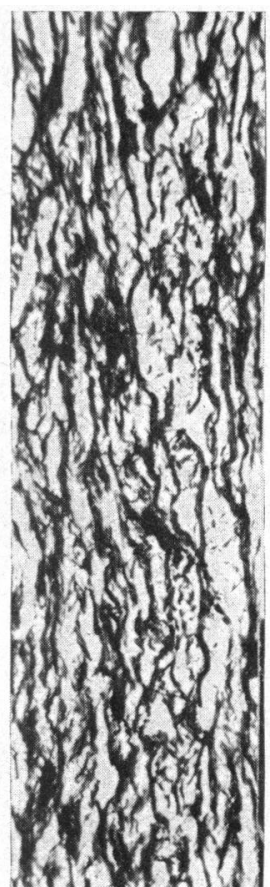

(D)

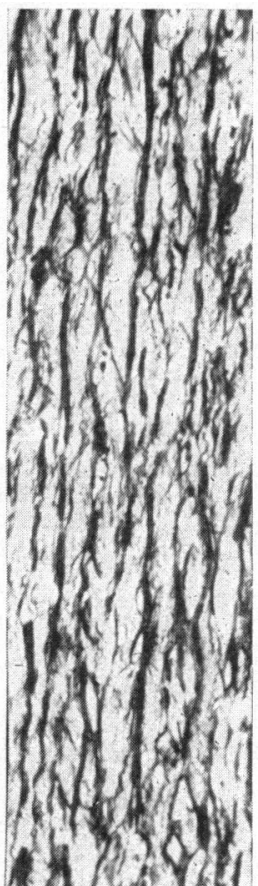

(E)

Fig. 2.-Transverse sections of pulmonary trunks from five Peruvian Indians who were born and spent most of their lives at high altitude. All show an aortic configuration of elastic tissue. (Elastic-van Gieson. $\times 150$.) The ages, in years, of the five subjects are: (A) 18 ; (B) 25 ; (C) 23 ; (D) 23 ; (E) 24 .

age-group the fibrils took up the stains for elastic tissue less well and stained grey rather than black.

Five subjects showed an undoubted "aortic" pattern of elastic tissue. Three of these were in people who had died in Lima but who had lived for a large part of their lives at high altitudes (Fig. 2). Two of the seven subjects who died at Cerro de Pasco also showed an aortic configuration of elastic tissue (Fig. 2). In these cases the pattern of the elastic fibrils was in striking contrast to that occurring in the adult pulmonary configuration. The fibrils formed a dense network; they tended to be long and uniform without the branching and clubbing of the adult pulmonary pattern; even more characteristically they tended to run parallel with one another, as in the aorta.

One of the subjects who died at Cerro de Pasco showed a pattern of elastic tissue which was transitional between an aortic and an adult pulmonary form (Fig. 3). The density of elastica was not great, and in places the fibrils formed an open network, but in other areas the fibrils tended to run parallel with one another to mimic an aortic structure. Since this was the only specimen that showed such a transitional pattern, and since it was thought to correspond more to the adult pulmonary than to the aortic type, the measurements made on it have, for the purpose of statistical analysis, been included in the adult pulmonary group.

The mean thickness of the fresh media of the pulmonary trunk measured in the present fashion was $1121 \mu$ (S.D. $=58 \mu)$. Age had no effect on this measurement. The thickness of the medias in the specimens with an aortic pattern of elastic tissue were $985,1220,1110,960$, and $1000 \mu$.

Extensibility. The general relation between length and tension was a curved one similar to that found in the previous studies (Harris et al., 1965a, b). Among the subjects in whom the pattern of elastic tissue in the pulmonary trunk was of the normal adult type, there was a general decrease in extensibility with an increase in age (Fig. 4).

In order to determine the average values shown in Fig. 4 a separate length-force curve was drawn for each case, and the degree of extension for a given extensile force was estimated by interpolation. Average values of extension were then calculated for a series of common extensile forces.

The same data could be used to examine the relation between the age of subject and the degree of extension for a given extensile force. As in the 


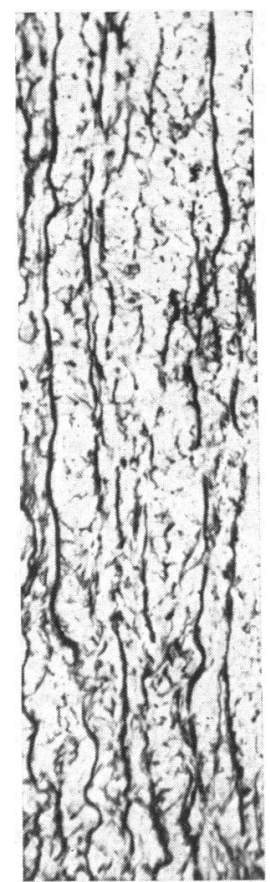

Fig. 3.-Transverse section of the pulmonary trunk from a Peruvian Indian aged 45 years who was born and spent his entire life at high altitude in the Andes. The configuration of the elastica is of transitional type. (Elastic-van Gieson. $\times 150$.

previous study (Harris et al., 1965a), the relation between the age and the logarithm of the degree of extension approximated to a straight line, the slope and intercept of which varied with the extensile force. Thus, for a particular extensile force, the relation between age and extension could be given by:

$$
\Delta 1=a .10^{b \times a g e}
$$

when $\Delta l$ is the percentage increase in length and $a$ and $b$ are constants.

The values of $a$ and $b$ varied according to the extensile force. Some were calculated from regression equations relating $\log _{10} \Delta 1$ to age. In the previous study, a simple mathematical relation was found to exist between these constants and the magnitude of the extensile force. In the present study, no such simple formula could be found to fit the data, and the relations between $a$ and $b$ and the magnitude of the extensile force are presented graphically (Fig. 5 and 6).

Thus, for a given extensile force, values of $a$ and $b$ could be obtained. When these were introduced into equation (1) together with the age of the subject, the expected degree of extension could be

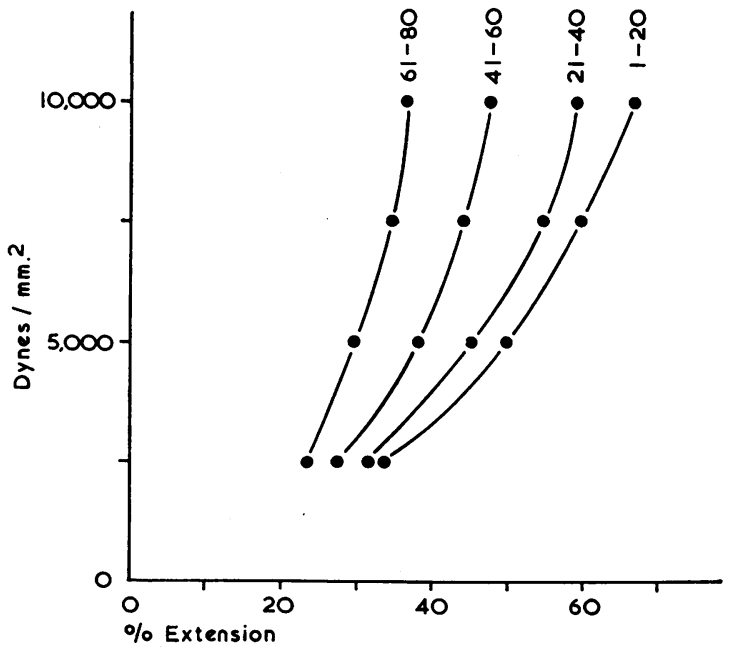

FIG. 4.-Average values for the extensibility of the pulmonary trunk in different age-groups.

calculated. Fig. 7 shows the observed degree of extension plotted against that predicted from equation (1) in $\mathbf{4 2}$ specimens with a normal adult pattern of elastic tissue. The regression equation for observed $(y)$ on predicted $(x)$ values for the percentage extension is as follows:

$$
y=-0.65+1 \cdot 034 x
$$

and the standard error of the estimate is 6.0 .

Figure 8 shows the observed degree of extension plotted against that predicted from equation (1) in

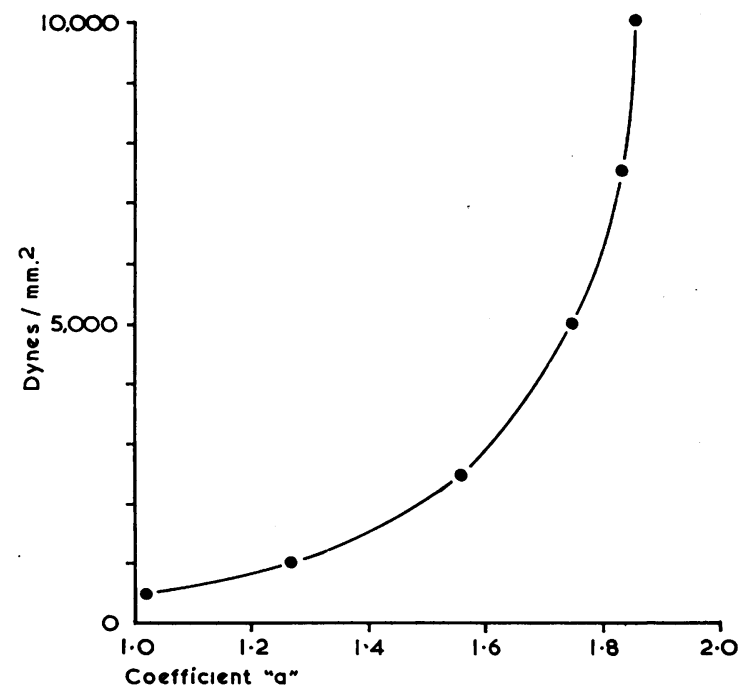

Fig. 5.-The relation between the extensile force and coefficient " $a$ ". 


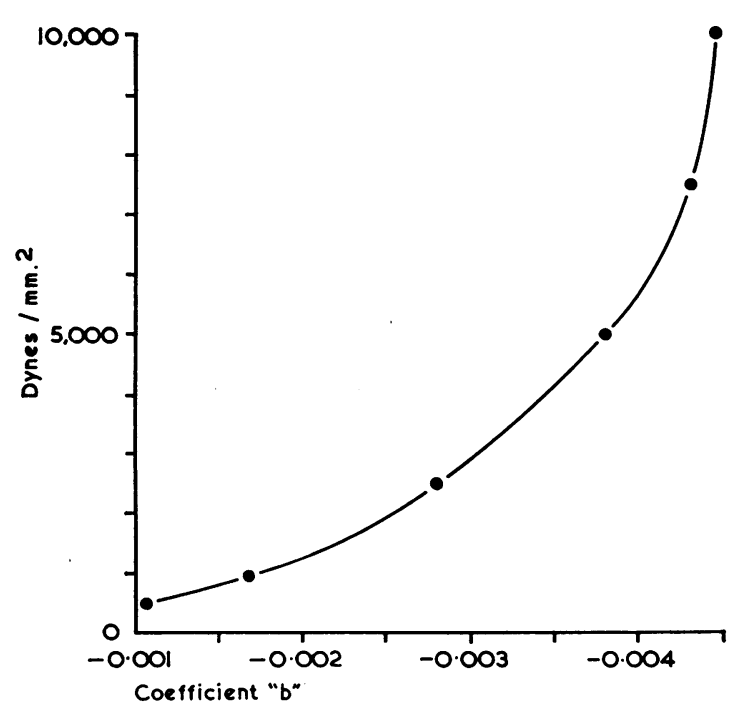

FIG. 6.-The relation between the extensile force and coefficient " $b$ ".

the five specimens showing an "aortic" type of elastic tissue. The line of the normal regression equation is shown together with lines at a distance of twice the standard error. It is evident that the extensibility of these specimens is less than normal, and this difference would be expected to arise by chance less often than once in a thousand times.

Chemical Analyses. Collagen was estimated in 28 specimens which had a normal adult pattern of elastic tissue and in 3 specimens which had an "aortic" pattern. In the group with the normal adult pattern, the average content of collagen was 31 per cent of the weight of dry tissue $(S D=5.0 \%)$,

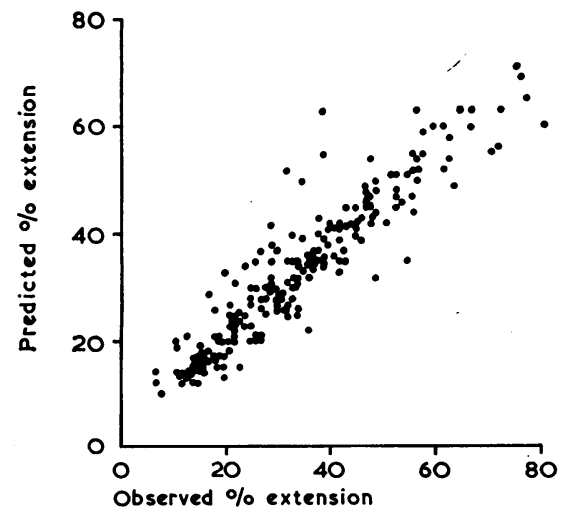

FIG. 7.-The relation between the predicted and observed values for percentage extension.

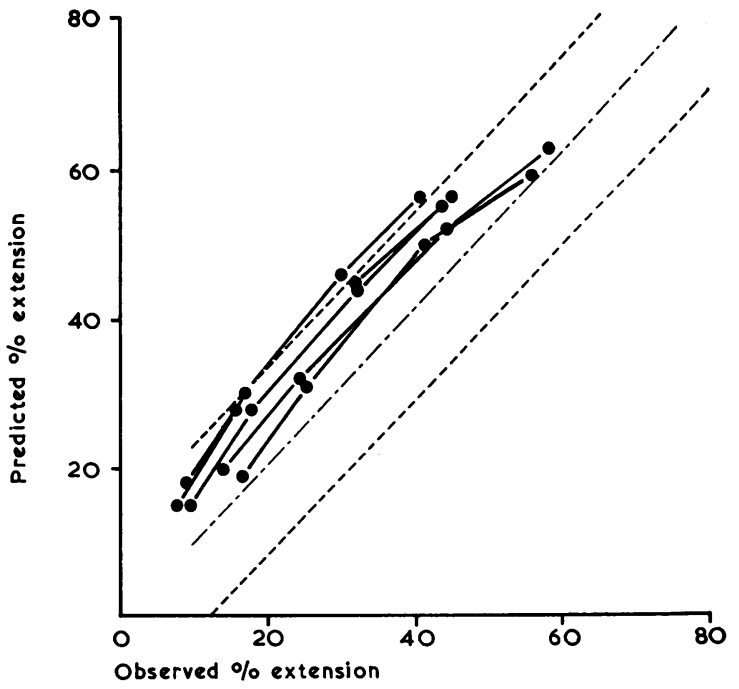

Fig. 8. -The relation between the observed degree of extension and that predicted from equation (1) in the five subjects with an aortic pattern of elastic tissue. The "normal" regression line of equation (2) is shown, together with lines at a distance of twice the standard error of the estimate.

while the average age was 46.4 years. There was a slight tendency for the content of collagen to decrease with age (Fig. 9), but this was readily attributable to chance $(r=-0.3288 ; p>0.05)$. In the three specimens with an aortic pattern of elastic tissue, the content of collagen was $31 \cdot 7,28 \cdot 3$, and 37.9 per cent of the weight of dry tissue, which did not differ from the group with a normal adult pattern (Fig. 9).

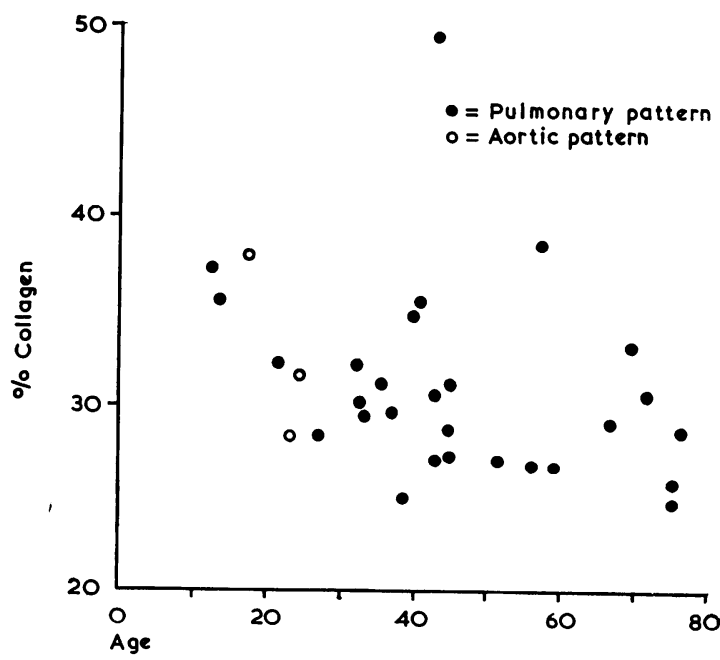

FIG. 9.-The relation between age and the content of collagen (\% dry weight). 


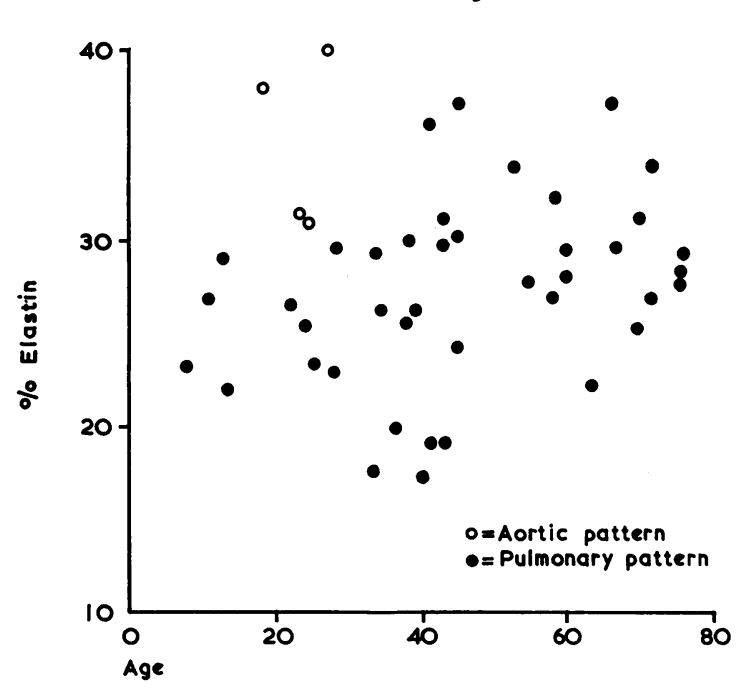

Fig. 10.-The relation between age and the content of elastin ( $\%$ dry weight).

Elastin was estimated in 41 specimens which had a normal adult pattern of elastic tissue and in 4 specimens which had an "aortic" pattern. In the group with the normal adult pattern, the average content of elastin was 27.2 per cent of the weight of dry tissue ( $\mathrm{SD}=4.8 \%$ ), while the average age was 45.4 years. There was a tendency for the content of elastin to increase with age (Fig. 10), which was unlikely to have arisen by chance $(r=+0.3382$;

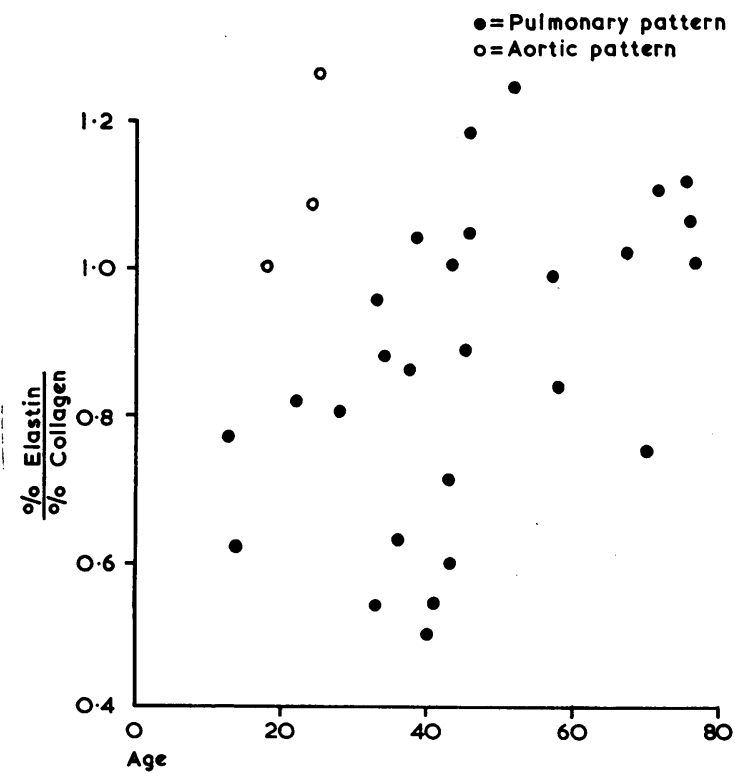

FIG. 11.-The relation between age and the ratio of elastin to collagen. $0.05>p>0.02$ ). In the four specimens with an "aortic" pattern of elastic tissue, the content of elastin was $40 \cdot 0,31 \cdot 4,31 \cdot 0$, and 37.9 per cent of the weight of dry tissue, the average age being 22.5 years. As will be seen from Fig. 10, these figures are inappropriately high for the age, and this difference is unlikely to have arisen by chance more often than once in a thousand times.

Both collagen and elastin were measured in 27 specimens with a normal adult pattern of elastic tissue (Fig. 11). As would be expected from the preceding figures, there was a positive correlation between the ratio of elastin to collagen and the age of the subject $(r=+0.4973 ; 0.01>p>0.005)$. Measurements of both collagen and elastin were available in 3 specimens with an "aortic" pattern of elastic tissue. In these specimens the ratio of elastin to collagen (Fig. 11) was inappropriately high for the age, a difference that is unlikely to have arisen by chance more often than once in a thousand times.

\section{Discussion}

Among those specimens with a normal adult histological pattern of elastic tissue, the extensibility was similar to that found previously (Harris et al., 1965a). The observation that extensibility decreases with age was also confirmed. Roach and Burton (1959) found a similar change with age in the human external iliac artery. These authors (Roach and Burton, 1957) ascribed the curved shape of the force-extension diagram of the walls of blood vessels to a shorter relaxed length of the elastic tissue relative to that of collagen. Thus, the first and more horizontal part of the curve was thought to be due almost entirely to the extension of elastic tissue, while the gradual involvement of the collagenous fibres coincided with the development of the more vertical later part of the curve.

Looked at from this point of view, the slope of the curve in its early stages should be related to the proportion of elastin (Roach and Burton, 1959). Fig. 12 plots the degree of extension at a relatively low extensile force of 2500 dynes $/ \mathrm{mm}^{2}$ against the age of the subject. Among the specimens with an adult pulmonary pattern of elastic tissue, the degree of extension falls with age $(r=-0.5078 ; p<0.001)$, and this is consistent with the increasing proportion of elastin found chemically both in the present study (Fig. 10) and by other authors (Lansing, Rosenthal, and Alex, 1950). When the degree of extension at 2500 dynes $/ \mathrm{mm}^{2}$ is plotted against the proportion of elastin (Fig. 13) there is a negative correlation $(r=-0.5024 ; 0.005>p>0.001)$, and this includes the specimens with an aortic pattern 


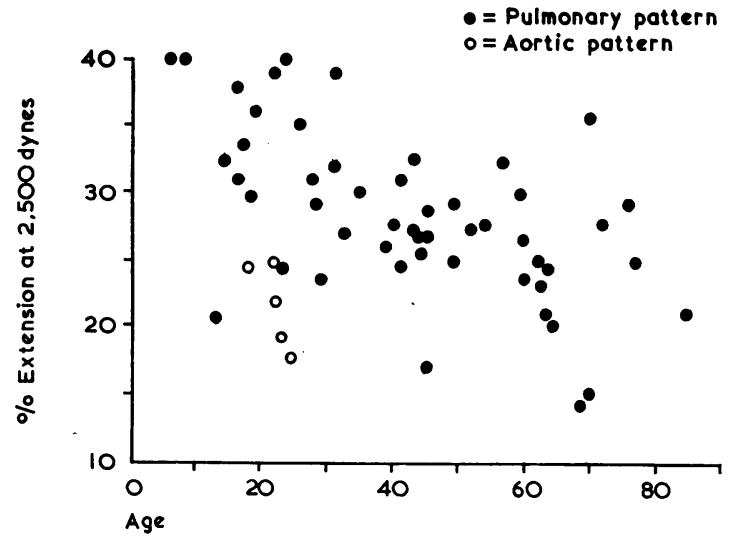

FIG. 12.-The relation between age and the degree of extension at 2500 dynes/mm. ${ }^{2}$.

of elastic tissue in whom the proportion of elastin does not conform with the age of the subject.

Inspection of the curves in Fig. 4 suggests that, while the slope of the initial part of the curve becomes steeper with an increasing age, there is at the same time an increasing steepness of the later part of the curve which proceeds at an even greater rate. Thus, the relation between force and extension became more curved with an increasing age. A simple measurement of the curvilinearity of the relation may be made by calculating the ratio of the degree of extension at 10,000 dynes $/ \mathrm{mm}^{2}$ to that at 5000 dynes $/ \mathrm{mm}^{2}$ Such a ratio would decrease from a value of 2.0 in the presence of a rectilinear relation to a theoretical minimum of 1.0 as the curvilinearity increased. When the ratio is plotted

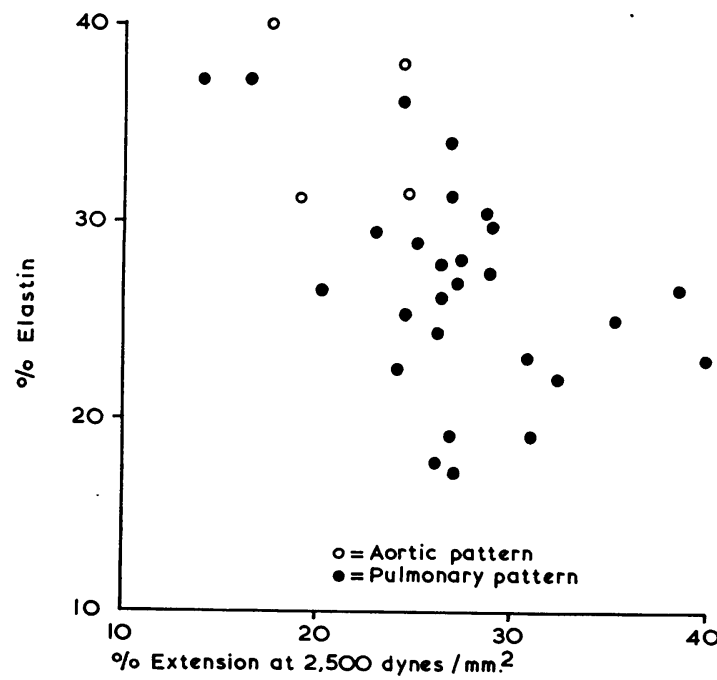

Fig. 13.-The relation between the degree of extension at 2500 dynes $/ \mathrm{mm} .^{2}$ and the content of elastin ( $\%$ dry weight).

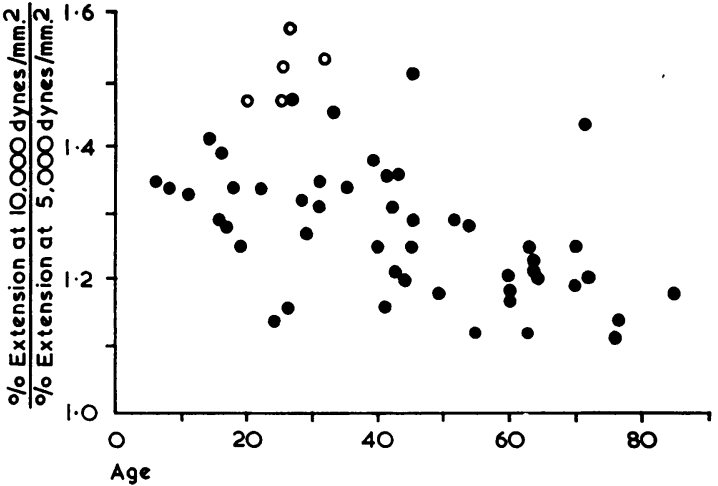

FIG. 14.-The relation between age and the ratio of extensibilities at 10,000 dynes $/ \mathrm{mm} .^{2}$ and 5000 dynes $/ \mathrm{mm}^{2}$.

against the age of the subject (Fig. 14) there is a negative correlation $(r=-0.4976 ; p<0.001)$ among those specimens with a pulmonary pattern of elastic tissue.

A decreased extensibility during the later portion of the force: extension diagram has been taken by Roach and Burton (1959) as evidence of an increased proportion of collagen. A similar explanation was previously put forward to explain the change in the force:extension curve of the pulmonary trunk which occurred with age (Harris et al., 1965a). This view appeared consistent with the increase in the amount of red-staining material visible under the microscope after staining with van Gieson's reagents. Yet, in the present studies, the chemical estimations failed to show any increase in the content of collagen with ageing. It is possible that ageing causes an alteration in the tinctorial properties of collagen. While, however, this would resolve the discrepancy between the microscopical and chemical findings, it leaves unexplained the decrease in extensibility with age. Moreover, it seems that a similar discrepancy occurs in the pulmonary arteries of children with tetralogy of Fallot, which show an increased amount of collagen under the microscope (Heath et al., 1959), which is not apparent on chemical analysis (Farrar, Blomfield, and Reye, 1965). One theoretical possibility is that the collagen becomes less extensible with age. There seems to be an increase in the bulk modulus of collagen with age (Banfield, 1956; Kohn and Rolleson, 1958), and this apparently applies to the perivascular connective tissue of the lung (Kohn, 1959). On the other hand, Young's modulus of the tendon from the human diaphragm has been found to become slightly lower in old age (Kohn and Rolleson, 1959). Another possible explanation is that, with an increasing age, the collagenous fibres become straighter. 
In this way they start to become stretched at an earlier point in the force:extension diagram. There may be more subtle alterations in the disposition of the fibres. Both collagenous and elastic fibres form a complex meshwork, the extensile properties of which are likely to depend not only on the simple linear extensibility of the individual fibres but also on their resistance to lateral deformation and on the geometry of the woven pattern itself. In this context it may be noted that Roach and Burton (1959) found that at no time did the extensibility of the wall of the human external iliac artery diminish to the extent that might be expected from a knowledge of Young's modulus for collagen.

In the specimens with an aortic pattern of elastic tissue, the chemical estimations revealed an abnormally high proportion of elastin for the age of the subject (Fig. 10). This was consistent with the microscopical appearances. Farrar et al. (1965) have recently shown a similar increase in the proportion of elastin in the pulmonary trunks of children when the aortic pattern of elastic tissue was due to congenital heart disease. The degree of extension at an extensile force of 2500 dynes $/ \mathrm{mm}$. $^{2}$ (Fig. 12) was abnormally low for the age in the group of specimens with an aortic pattern of elastic tissue $(p<0.001)$, and this is consistent with the presence of excess elastin (Fig. 13).

The proportion of collagen was normal in the groups with an aortic pattern of elastic tissue (Fig. 9), so that the ratio of elastin to collagen was abnormally high (Fig. 11). This may possibly explain the abnormally high values of the ratio of extension at 10,000 dynes $/ \mathrm{mm}^{2}{ }^{2}$ to that at 5000 dynes $/ \mathrm{mm} .^{2}$ in these specimens $(p<0.001)$.

Such observations suggest an abnormally low degree of extensibility in the early stages of extension, which is not immediately apparent from Fig. 8. As discussed elsewhere (Harris et al., 1965b), this is simply because Fig. 8 shows the absolute deviation of the observations from the predicted value rather than the percentage discrepancy. When the degree of extension is expressed as a percentage of that predicted (Fig. 15), 4 out of 5 specimens show a maximal diminution in extensibility at low degrees of extension. Fig. 16 expresses in the same fashion the average for these 5 specimens and compares it with the average of 2 specimens with an aortic pattern of elastic tissue due to congenital heart disease taken from a previous study (Harris et al., 1965b). Both groups show a maximal diminution in extensibility at low degrees of extension.

It is of interest to contrast these two groups of observations with those from a group of subjects with tetralogy of Fallot taken from a previous study

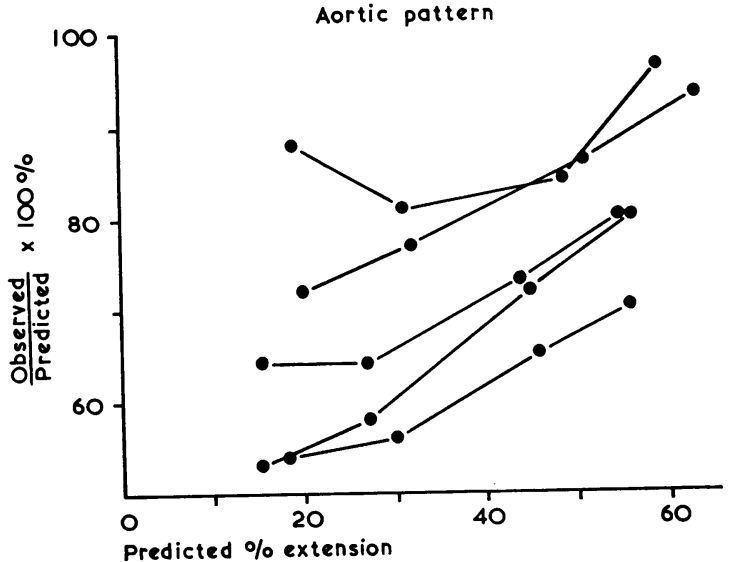

FIG. 15.-The relation between the degree of extension and the degree of extension expressed as a percentage of that predicted in the subjects with an aortic pattern of elastic tissue.

(Harris et al., 1965b). In such subjects there is an abnormally low proportion of elastic tissue and an abnormally high proportion of collagen visible under the microscope. Fig. 16 shows that, in these specimens, the ratio of observed to predicted extensibility

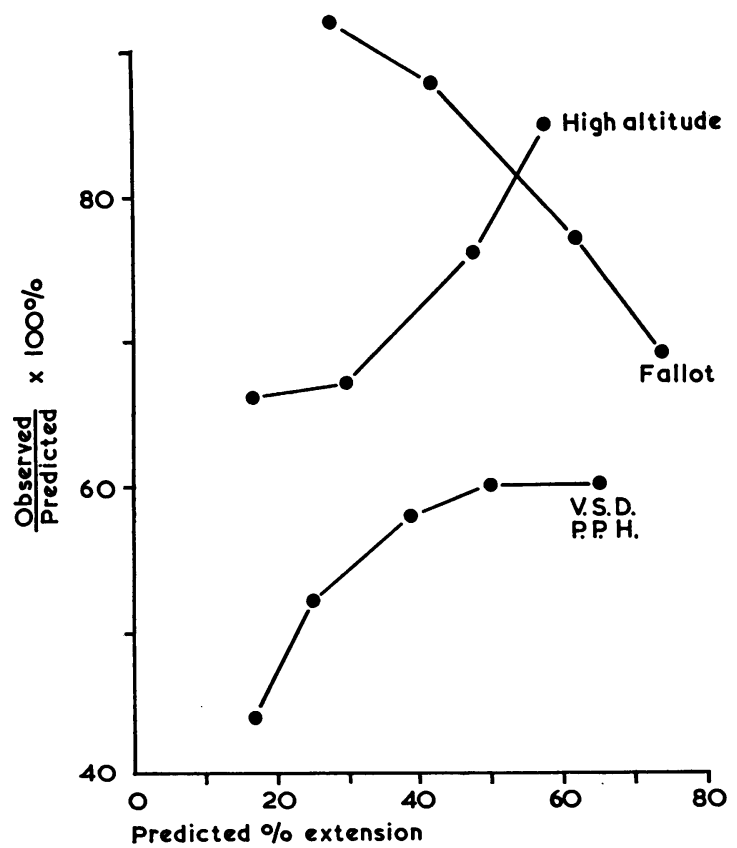

Fig. 16.-The relation between the degree of extension and the degree of extension expressed as a percentage of that predicted. Average values of three groups are shown. (i) Aortic elastic pattern due to high altitude; (ii) aortic elastic pattern due to ventricular septal defect and primary pulmonary hypertension; (iii) tetralogy of Fallot. 
becomes lower with an increasing degree of extension, which is the opposite of the two groups with an increased content of elastin.

\section{SUMMARY}

The histology, physical extensibility, and chemical composition of the media of the pulmonary trunk has been studied after death in people who had lived at sea-level and at high altitude in Peru.

Among those who had lived at sea-level and who had a normal pattern of elastic tissue there was a gradual decrease in the physical extensibility of the wall of the pulmonary trunk with increasing age and a gradual increase in the concentration of elastin.

Some of those native to high altitudes showed a pattern of elastic tissue similar to that found in the fotal pulmonary trunk or in the aorta, an abnormality which suggests the presence of pulmonary hypertension from birth. The extensibility of such specimens was inappropriately low for the age of the subject and the content of elastin inappropriately high. The decrease in extensibility was proportionally greatest at light loads where the effects of elastin are thought to predominate.

Neither age nor altitude appeared to have any influence on the content of collagen.

\section{REFERENCES}

Banfield, W. G. (1956). Age changes in the swelling capacity of the human Achilles tendon. F. Geront., 11, 372.

Farrar, J. F., Blomfield, J., and Reye, R. D. K. (1965). The structure and composition of the pulmonary circulation in congenital heart disease. F. Path. Bact., 90, 97.

Harris, P., and Heath D. (1962). The Human Pulmonary Circulation. E. and S. Livingstone, Edinburgh.

,-- , and Apostolopoulos, A. (1965a). Extensibility of the human pulmonary trunk. Brit. Heart. F., 27, 651 .

$\longrightarrow,-$, and $-(1965 \mathrm{~b})$. Extensibility of the pulmonary trunk in heart disease. Brit. Heart $\mathcal{F}$., 27, 660 .

Heath, D., DuShane, J. W., Wood, E. H., and Edwards, J. E. (1959). The structure of the pulmonary trunk at different ages and in cases of pulmonary hypertension and pulmonary stenosis. $\mathcal{F}$. Path. Bact., 77, 443.

Kohn, R. R. (1959). Age and swelling in acid of perivascular connective tissue in human lung. f. Geront., 14, 16.

—, and Rollerson, E. (1958). Relationship of age to swelling properties of human diaphragm tendon in acid and alkaline solutions. F. Geront., 13, 241.

$\longrightarrow$, and - (1959). Effect of age and heat on human collagenous tissue. Arch. Path., 68, 316.

Lansing, A. I., Rosenthal, T. B., and Alex, M. (1950). Significance of medial age changes in the human pulmonary artery. F. Geront., 5, 211.

Lowry, O. H., Gilligan, D. R., and Katersky, E. M. (1941). The determination of collagen and elastin in tissues, with results obtained in various normal tissues from different species. F. biol. Chem., 139, 795.

Peñaloza, D., Sime, F., Banchero, N., and Gamboa, R. (1962). Pulmonary hypertension in healthy man born and living at high altitudes. Med. thorac. (Basel), 19, 449.

Roach, M. R., and Burton, A. C. (1957). The reason for the shape of the distensibility curves of arteries. Canad. $\mathcal{F}$. Biochem., 35, 681.

- , and - (1959). The effect of age on the elasticity of human iliac arteries. Canad. F. Biochem., 37, 557.

Saldaña, M., and Arias-Stella, J. (1963). Studies on the structure of the pulmonary trunk. II. The evolution of the elastic configuration of the pulmonary trunk in people native to high altitudes. Circulation, 27, 1094.

Wagenvoort, C. A., Heath, D., and Edwards, J. (1964). The Pathology of the Pulmonary Vasculature. Charles C. Thomas, Springfield, Illinois. 